\title{
Autoetnografía y Nuevas Formas Literarias para las Ciencias Sociales. Presentación de la Carpeta
}

\author{
Joel Feliu i Samuel-Lajeunesse y Adriana Gil Juárez \\ Universitat Autònoma de Barcelona \\ joel.feliu@uab.cat adriana.gil@uab.cat
}

Se presenta en esta carpeta el producto de dos seminarios que tuvieron lugar en el contexto del Máster en Investigación en Psicología Social y del Doctorado en Psicología Social de la Universitat Autònoma de Barcelona. Estos seminarios se propusieron investigar sobre el género científico, en tanto que género literario, para experimentar con el potencial de algunas de las propuestas más recientes que se han realizado en escritura para las ciencias sociales, especialmente en el contexto de la segunda edición del Handbook of Qualitative Research, editado por Norman Denzin y Yvonna Lincoln.

El primer trabajo de esta carpeta consiste en una presentación del género autoetnográfico, aunque sus implicaciones no solamente se dirigen a la autoetnografía, en él se analizan sus características, su interés y sus dificultades.

Seguidamente, se presentan los trabajos generados en el marco del seminario sobre investigación de las nuevas tecnologías. En este seminario se retomó la cuestión siempre insinuada, apenas empezada y nunca acabada de la relación entre emociones, consumo, afectividad y virtualidad, pero centrándose específicamente en las nuevas tecnologías. La pregunta que orientó el seminario fue: ¿Cómo enfrentar una investigación que implique las nuevas tecnologías en todas sus dimensiones (desde las virtuales hasta las que afectan los espacios físicos en que tiene lugar su consumo)? Con este fin se propuso la realización de autoetnografías tecnológicas a los asistentes en las que relataran su entrada y apropiación personal del mundo de las nuevas tecnologías. En esta carpeta se presentan tres de estas autoetnografías biográficas.

Más adelante, se presentan los trabajos generados en el marco de un seminario sobre la condición de la escritura en ciencias sociales. En este seminario se planteó la problemática y los debates entorno a la creación de conocimiento mediante la escritura. Ciertamente, el momento de escribir en el proceso de la investigación es un buen momento para preguntarse si lo que escribimos ya lo sabíamos antes de escribirlo y si el contenido es tan independiente de la forma como el formato científico sugiere. Aunque en la búsqueda de nuevas formas literarias que cuestionen los efectos de poder habituales al género científico, no debe perderse de vista el objetivo final de la producción de conocimiento en forma de reflexión de la sociedad sobre sí misma, que es a lo que se dedican en general las ciencias sociales. 
Los cinco trabajos que conforman el segundo bloque de esta carpeta, presentan experiencias tan variadas como una obra de teatro, una novela, un guión para un programa de radio, una autoetnografía y un monólogo.

\section{Formato de citación}

Feliu, Joel y Gil, Adriana, Nombre (2007). Autoetnografía y Nuevas Formas Literarias para las Ciencias Sociales. Presentación de la Carpeta. Athenea Digital, 12, 260-261. Disponible en http://psicologiasocial.uab.es/athenea/index.php/atheneaDigital/article/view/446

Joel Feliu I Samuel-Lajeunesse. Es doctor en Psicología Social y profesor titular del Departamento de Psicología Social de la Universitat Autònoma de Barcelona. Investigador de los grupos JovenTIC y GESCyT. Destacan entre sus publicaciones recientes la coedición con Adriana Gil de Psicología Económica y del Comportamiento del Consumidor. Barcelona: Editorial UOC, 2004; y su participación en el libro de Gil y Vall-llovera Jóvenes en Cibercafés: La dimensión física del futuro virtual. Barcelona: Editorial UOC, 2006.

Adriana Gil Juárez. Magíster y Doctora en Psicología Social. Actualmente es Profesora Asociada del Departamento de Psicología Social de la Universitat Autònoma de Barcelona (UAB). Es directora del grupo de investigación JovenTIC (http://psicologiasocial.uab.es/JovenTIC) que estudia las dinámicas de consumo de las TIC en jóvenes y su relación con sus procesos identitarios. Algunos de sus últimos libros son: (coord.) Tecnologías Sociales de la Comunicación. Barcelona: Editorial UOC, 2005; con Vall-llovera, M. Jóvenes en Cibercafés: la dimensión física del futuro virtual. Barcelona: Editorial UOC, 2006; El consumidor. Colección Quiero Saber. Barcelona: Editorial UOC, 2007; con Vida, T. Los Videojuegos. Colección TIC.Cero. Barcelona: Editorial UOC (en prensa).

Este texto está protegido por una licencia Creative Commons.
Usted es libre de copiar, distribuir y comunicar públicamente la obra bajo las
siguientes condiciones:
Reconocimiento: Debe reconocer y citar al autor original.
No comercial. No puede utilizar esta obra para fines comerciales.
Sin obras derivadas. No se puede alterar, transformar, o generar una obra
derivada a partir de esta obra.
\[ \underline{\text { Resumen de licencia }} \]
Texto completo de la licencia

